Pferdeheilkunde 3 (1987) 4 (Juli) 203-212

\section{Die Röntgenologie der Halswirbelsäule beim Pferd}

\author{
Katherine E. Whitwell und Sue Dyson
}

Pathology Unit und Equine Clinical Unit, Animal Health Trust

\section{Einleitung}

Die Indikation für eine Röntgenuntersuchung der Halswirbelsäule beinhaltet Ataxie, abweichende Kopf-Hals-Haltung, Muskelatrophie, Schwellungen im Halsbereich, steifen Gang oder Schmerzen, Unvermögen zu stehen und gelegentlich Lahmheit der Vordergliedmaßen.

Dieser Artikel hat das Ziel, einen Leitfaden zu erstellen für das röntgenologische Erkennen von Halswirbelsäulenschäden und deren Einschätzung hinsichtlich einer möglichen klinischen Bedeutung.

Es ist wichtig, daß andere klinische und labordiagnostische Untersuchungen miteinbezogen werden, wenn es darum geht, die Bedeutung der röntgenologischen Befunde der speziellen klinischen Fragestellung zuzuordnen. Eine Reihe normaler Halsaufnahmen und scharfer Halswirbelaufnahmen ist für den beabsichtigten $\mathrm{Zweck}$ unumgänglich.

\section{Röntgentechnik}

Diese Arbeit beschränkt sich auf die Interpretation von Röntgenbildern, und die Myelographie wird hierbei nicht berücksichtigt. Am stehenden Pferd ist die Röntgenuntersuchung auf die seitliche Projektion beschränkt. Dies wird sowohl im Strahlengang von links nach rechts als auch umgekehrt erreicht.

Leicht schräge Röntgenbilder sind schwer interpretierbar, und zumeist ist nur die rein seitliche Röntgenebene wesentlich. Die Sedation des Pferdes mit Xylazin oder Detomidine kann nützlich sein, da sich das Pferd dann wenig bewegt und die Tendenz hat, Kopf und Hals zu senken.

Obwohl gute Aufnahmequalität im kranialen und mittle-

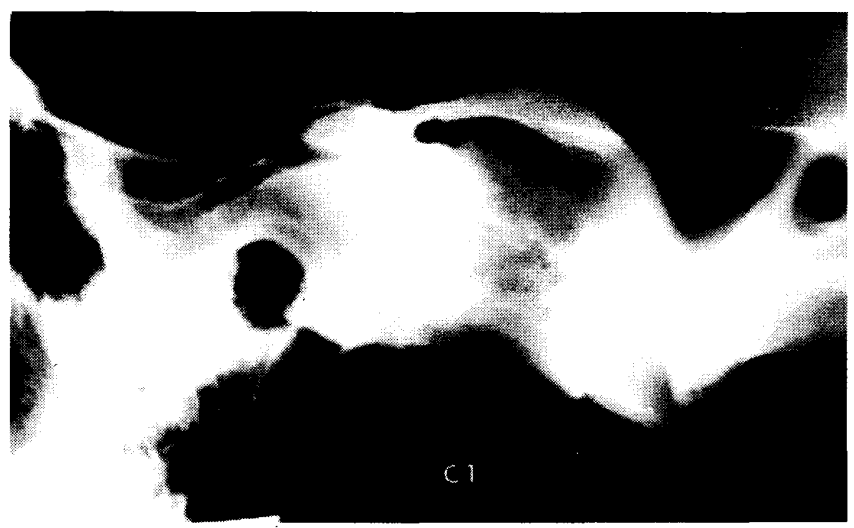

Abb. 1: Seitenansichten der kranialen Halswirbel $C 1$ bis $C 4$ beim ge sunden erwachsenen Pferd ren Halsbereich mit einem tragbaren Röntgengerät erreicht werden kann, ist für den kaudalen Halsbereich (7. Halswirbel: C7) eine stärkere Röntgenanlage unumgänglich, außer bei sehr kleinen Pferden. Ein Raster ist zwar für das kaudale Halsdrittel notwendig, aber es erübrigt sich weiter vorne. Bei Anwendung von hochempfindlichen Filmen, kombiniert mit Seltenen-Erden-Folien und Kreuzgitterraster, schwanken die Belichtungswerte von $65 \mathrm{kV}$ und $40 \mathrm{mAs}$ im vorderen Bereich des Halses bis zu $80 \mathrm{kV}$ und $65 \mathrm{mAs}$ im Halsbasisbereich eines $500 \mathrm{~kg}$ schweren Pferdes. Für eine bessere Orientierung im Halswirbelbereich sind große Kassetten $(35 \times 43 \mathrm{~cm})$ hilfreich, so daß 3 Halswirbel zumindest teilweise auf jede Kassette projiziert werden. Passives Abbeugen des Halses am anästhesierten Pferd verdeutlicht eine vorhandene Subluxationsstellung, die im seitlichen Strahlengang erkennbar ist. Dabei sollte die Zeitspanne des Abknickens des Endotrachealtubus möglichst kurz gehalten werden. Falls ventrodorsale Röntgenbilder erforderlich sind, sollte das Pferd in Allgemeinnarkose in Rükkenstellung gelagert werden. Selbst bei Hochleistungsröntgengeräten ist es schwierig, Aufnahmen guter Qualität kaudal von C 5 zu erhalten, da das Weichteilgewebe hier besonders stark ausgebildet ist.

\section{Röntgenanatomie}

Es gibt 7 Halswirbel (C1 bis C7), die von $\mathrm{C} 3$ bis $\mathrm{C} 7$ (Abb. 1, 2 und 3) kürzer und breiter werden. Der 3., 4. und 5. Wirbel sind in der Form ähnlich, während der Rest unverwechselbare Merkmale besitzt. Die beiden Seiten des Wirbelbogens (Wirbelbogenwurzeln) und der Wirbelkörper bilden das Foramen vertebrale, in dem das Rückenmark Platz findet (Abb. 4). Die Aneinanderreihung der Foramina vertebralia läßt so zusammen den Wirbelkanal entstehen. Der dorsoventrale (sagittale) Durchmesser des Wirbelkanals hat die Tendenz, im mittleren Halsbereich enger zu sein, verglichen mit den mehr kranialen oder kaudalen Abschnitten. Das Foramen vertebrale eines jeden Wirbels besitzt annähernd rechteckige Gestalt, und sein sagittaler Mindestdurchmesser läßt sich auf dem Röntgenbild direkt abmessen (Abb. 2). Dies ist durchaus sinnvoll, um die Halswirbel miteinander zu vergleichen. Vergleiche von Pferden untereinander sind aber auf Grund der durch die unterschiedliche Vergrößerung bedingten Fehlerquote nicht zu-

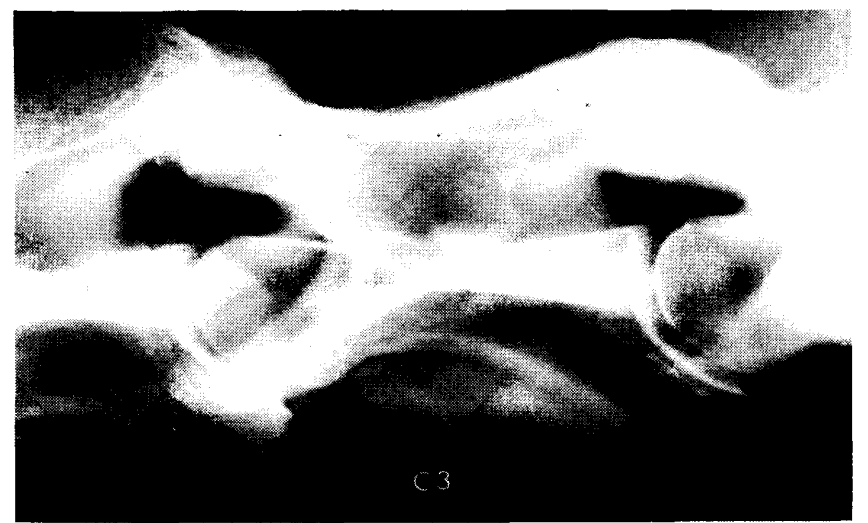




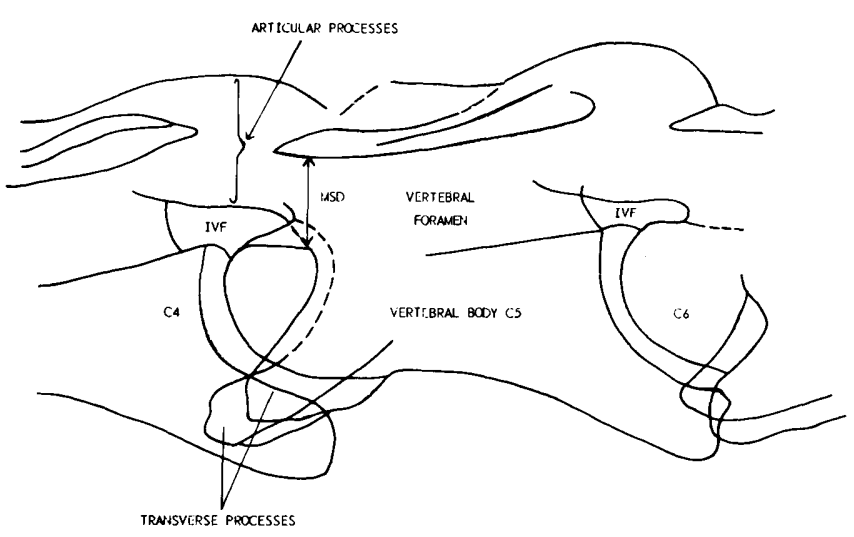

IVF - INTERVERTEBRAL FORNAEN

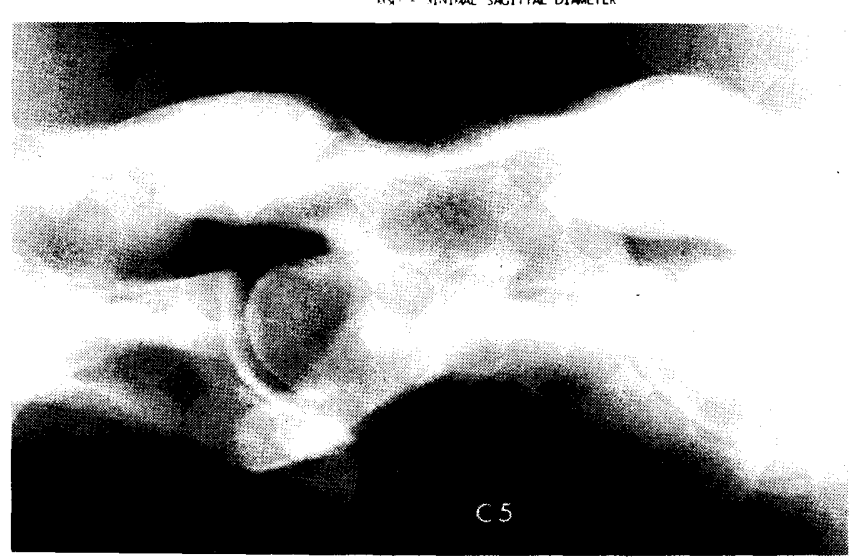

Abb. 2: Seitenansicht (röntgenologisch und schematisch) von C4 zu C6 beim gesunden erwachsenen Pferd.

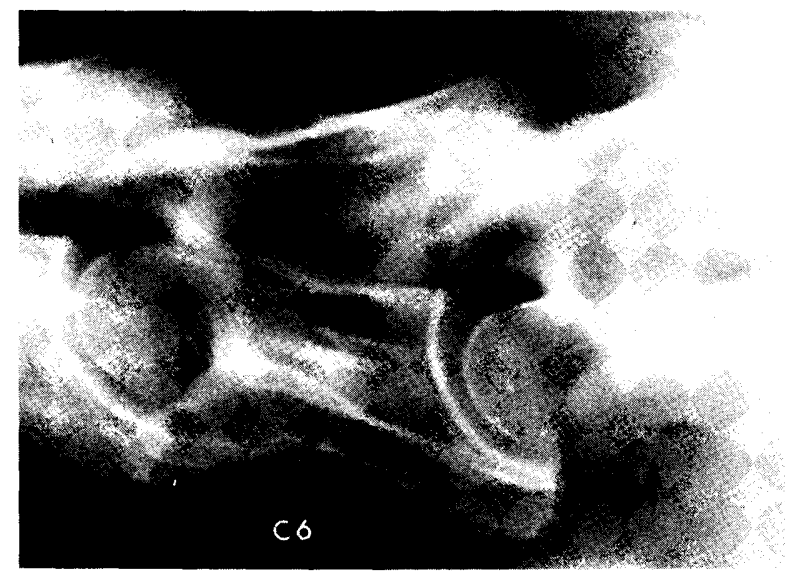

Abb. 3: Seitenansicht einschließlich $C 6$ und $C 7$ beim erwachsenen Pferd. Normale Konfiguration der ventralen Processus bei C6. Beachte den kleinen spondylotischen Vorsprung bei ventraler Ansicht von C7 (Pfeil).

lässig. Bei der Geburt sind von C 3 bis C7 die kraniale und kaudale Epiphyse auf beiden Seiten des Wirbelkörpers vorhanden (Abb. 5). Der Epiphysenfugenschluß (Synchondrose) ist ein schrittweiser Prozeß mit individueller Schwankungsbreite. Der Schluß der kranialen Wachstumsfuge beginnt ventral und stellt sich röntgenologisch komplett abgeschlossen im Alter von 2 Jahren dar. Die kaudale Wachstumsfuge bleibt bis zum 4. bzw. 5. Lebensjahr offen, ihr Zusammenschluß beginnt dorsal. Beim ausgewachsenen Pferd sind die Enden der Halswirbelkörper stark abgerun-

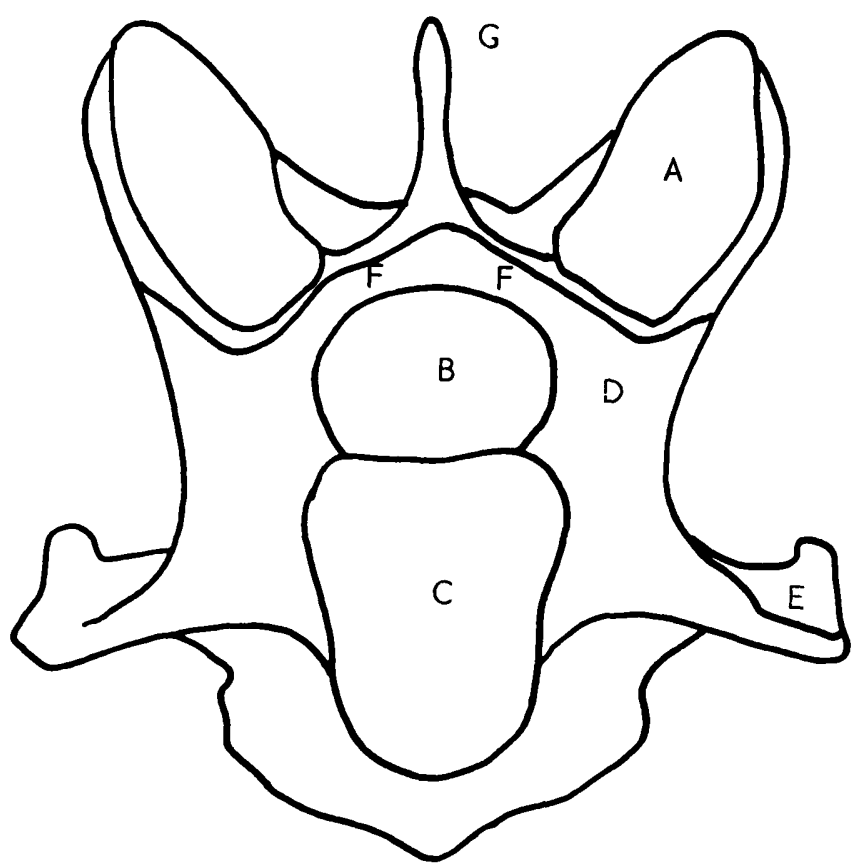

Abb. 4: Diagramm von C7 aus kaudokranialer Sicht: A - Gelenkfläche des kranialen Gelenkfortsatzes; B - Foramen vertebrale; C - Wirbelkörperkopf; D-lateraler Wirbelbogen oder Wirbelbogenwurzel; $\mathrm{E}$ Processus transversus; $F$-dorsale Knochenleiste, die den Wirbelbogen bildet; $\mathrm{G}$ - Dornfortsatz.

det, und ihre Kranialflächen sind konvex, während die Kaudalflächen konkav ausgebildet sind.

Von C 3 bis C7 besitzt jeder Wirbel einen kranialen und kaudalen Processus articularis, der sich an den Rändern der Wirbelkörper abzeichnet. Die Fortsätze der Processus articulares besitzen Gelenkflächen, die mit hyalinem Knorpel überzogen sind, um mit den sehr eng angelagerten benachbarten Wirbeln ein Gelenk bilden zu können. Die Halsnerven verlassen den Wirbelkanal durch die Foramina intervertebralia (Abb. 1 und 3). Der Atlas (C1) weist weder Wirbelkörper noch Gelenkfortsätze auf. Der Epistropheus (C2) hat getrennte Ossifikationszentren für Dens (Processus odontoidens), Kopf, Körper und die kaudale Epiphyse (Abb. 5). Ungefähr im Alter von 7 Monaten vereinigt sich der Dens mit dem Kopf. Der 6. Halswirbel unterscheidet sich vom C5 dadurch, daß er einen weiteren Processus transversus aufweist - eine zusätzliche ventrale Knochenlamelle bzw. einen Processus, der sich kaudal und ventral abzeichnet (Abb. 3). Als normale Variante können ein oder beide ventralen Processus, auf die ventrale Oberfläche des C7 transponiert, vorkommen. Wenn dies beiderseits vorkommt, hat die ventrale Oberfläche des C6 das gleiche röntgenologische Aussehen wie C5. Die ventralen Processus von $\mathrm{C} 6$ besitzen kleine Verknöcherungszentren an ihren kaudalen Begrenzungen, die röntgenologisch sichtbar sind und nicht mit Frakturlinien verwechselt werden sollten (Abb. 7). C7 besitzt einen kleinen, in der Höhe variierenden Processus spinosus.

In Ruhestellung besitzt die normale Halswirbelsäule eine SForm mit geschmeidigen Übergängen in der Winkelung zwischen den einzelnen Wirbeln, und zwar von dorsalkonvex im kranialen Bereich zu dorsalkonkav im kaudalen Halsabschnitt. Offensichtliche Hyperflexion bzw. Sublu- 


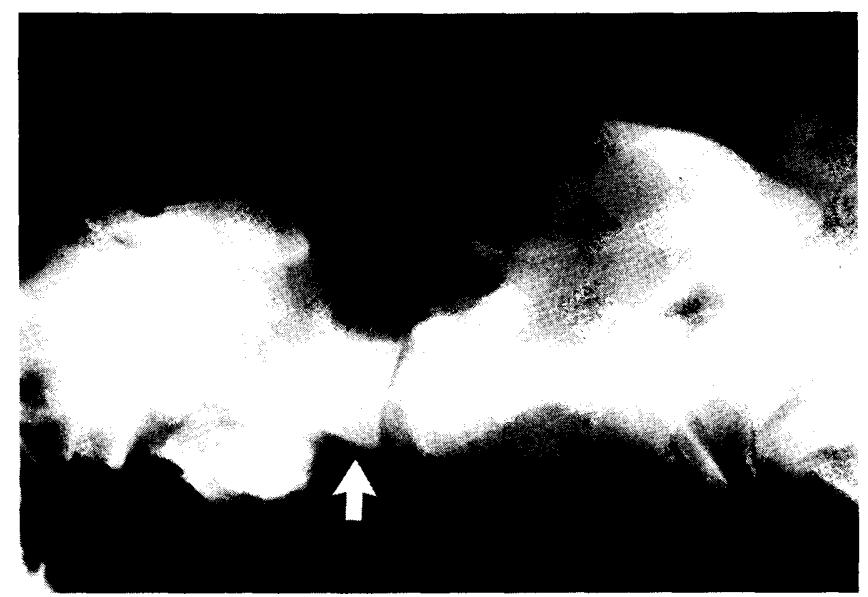

Abb. 5: Seitenansicht der kranialen Halswirbel eines 6 Wochen alten Volliblutfohlens. Beachte die getrennten Ossifikationszentren des Dens von C2 (weißer Pfeil). Die Wirbelkörper von C2 und C3 haben nicht vereinigte Epiphysen.

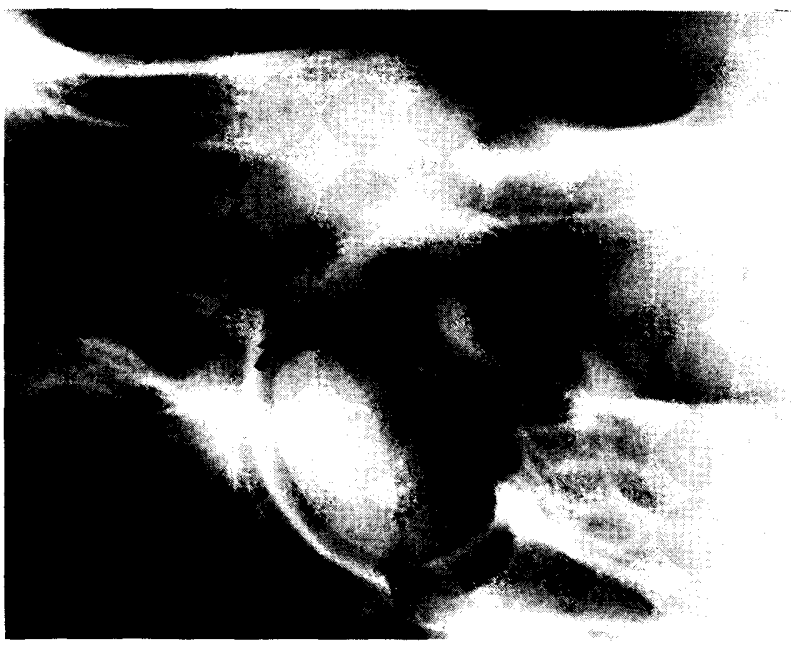

Abb. 6: Seitenansicht von C6 und C7. 7 Jahre alter Hunter-Wallach mit Ataxie seit 4 Wochen. Große Knochensäule (schwarzer Pfeit) am kranialen Gelenkfortsatz von C 7, teils das Foramen intervertebrale von C6 zu C 7 verlegend (offener Pfeil). Rückenmarksquetschung in diesem Bereich konnte bestätigt werden, verursacht durch eine Ausbeulung der Gelenkkapsel. Beachte die Transposition beider Knochenleisten von $\mathrm{C} 6$ zu C 7 (vgl. Abb. 3)

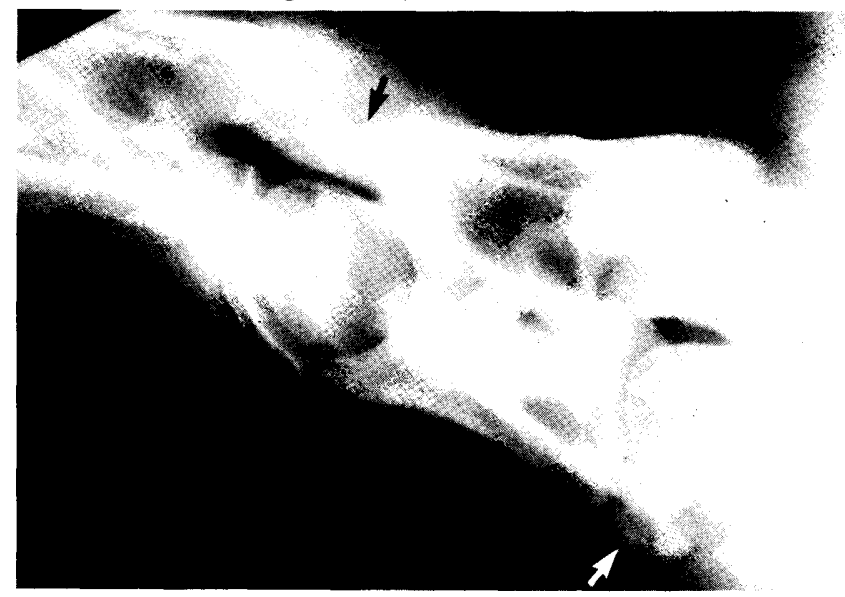

Abb. 7: Seitenansicht der kaudalen Halswirbelsäule eines ataktischen Vollblutiährlingsfohlens. Stenose des kranialen Orificiums von C6 (schwarzer Pfeil) läßt das Foramen vertebrale im Schnitt dreieckig erscheinen. Rückenmarkskompression wurde bei $C 5$ und $C 6$ bestätigt. Die getrennten Ossifikationszentren bei kaudaler Ansicht der ventralen Fortsätze von C6 (weißer Pfeil) sollten nicht mit Frakturen verwechselt werden.

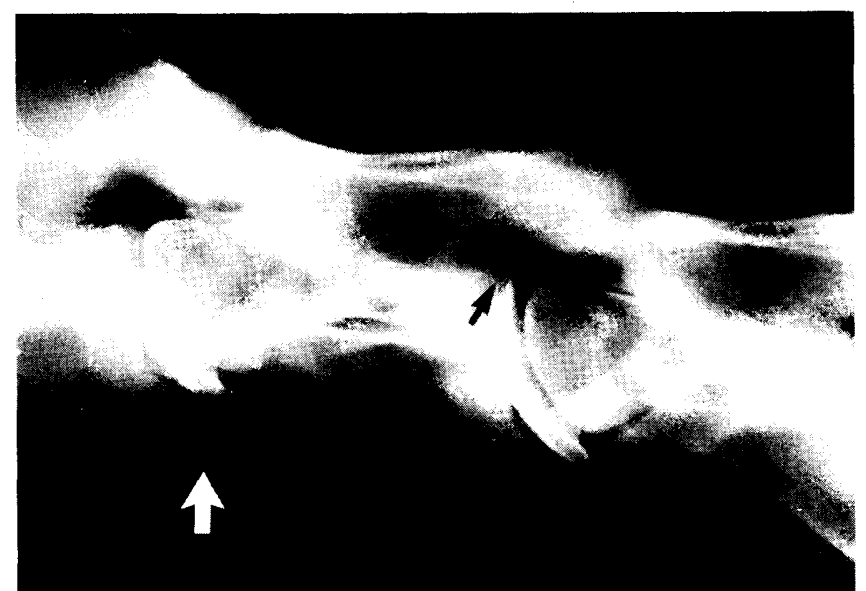

Abb. 8: Seitenansicht von $\mathrm{C} 2$ zu $\mathrm{C} 4$ bei einem ataktischen Jährling. Subluxationsstellung von $\mathrm{C} 2$ zu C 3 ist erkennbar (weißer Pfeil), aber die dorsoventralen Wirbelkanaldurchmesser waren nicht signifikant verengt. Rückenmarkskompression wurde im C6-C 7-Bereich in Verbindung mit einer ernsthaften Arthropathie festgestellt. Beachte die angemessene Erweiterung der kaudalen Epiphyse von C3 (schwarzer Pfeil).

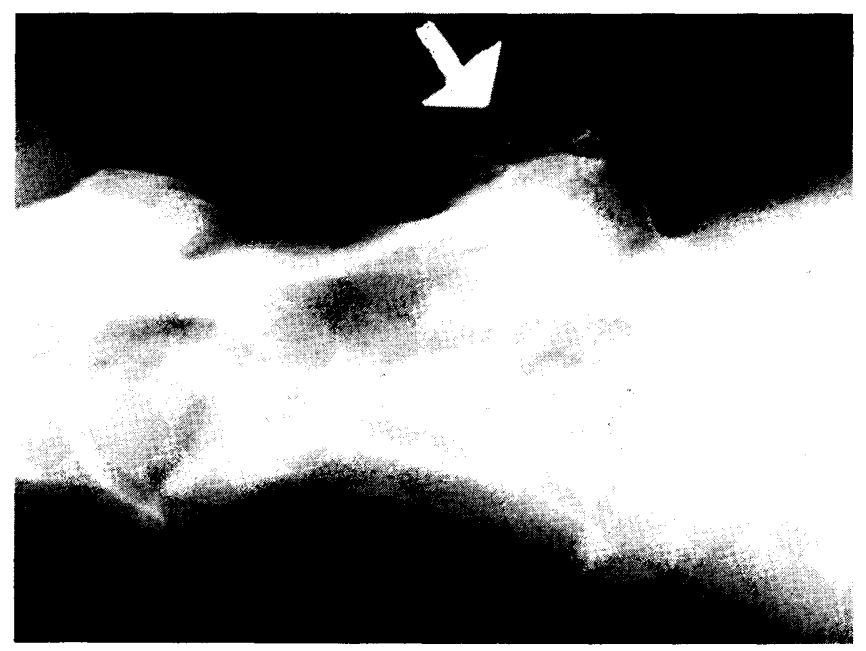

Abb. 9: Leicht schräge Seitenansicht der mittleren Halsregion eines erwachsenen Pferdes. Es ist eine diffuse Verkalkung im Weichteilgewebe vorhanden, die einer i. m. Injektion folgte. Die Röntgenverschattung entsteht durch teilweise Uberlagerung mit dem Gelenk von C 4 zu $\mathrm{C} 5$ und sollte nicht mit Wirbelschädigungen verwechselt werden.

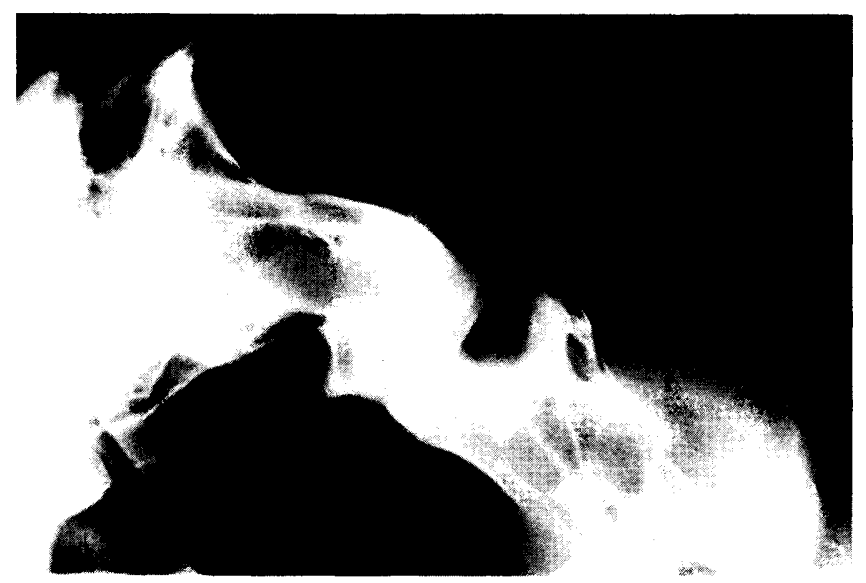

Abb. 10: Seitenansicht der kranialen Halswirbel eines Araberjährlings mit vollausgebildeter Ataxie und abnormalem Hervortreten der Atlasflügel. Der Atlas ist mit dem Hinterhauptsbein verschmolzen, und C2 ist mißgebildet - eine atlantookzipitale Achsenfehlstellung (vgl. mit Abb. 1 und 5) 


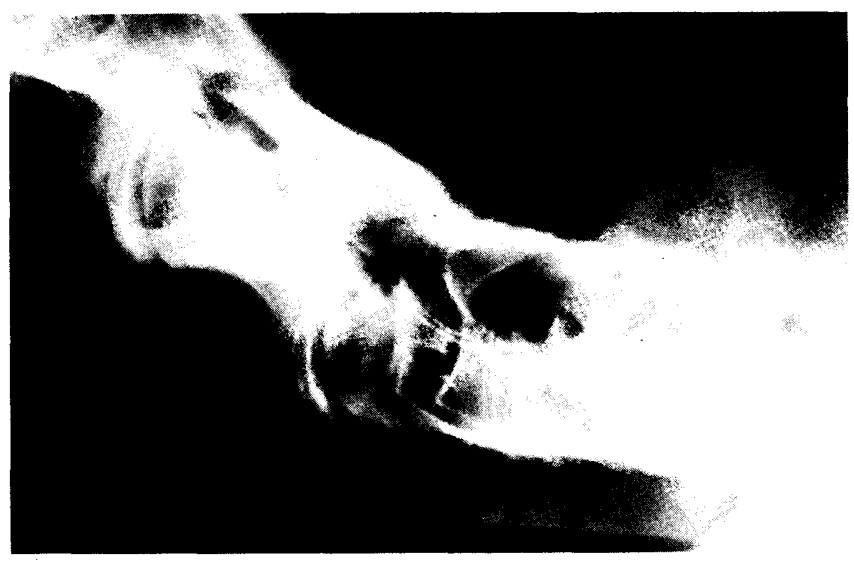

Abb. 11: Seitenansicht von $C 5$ bis $T 1$ bei einem 5 Monate alten Vollblutfohlen. C6, C7 und T 1 sind mißgebildet und verschmolzen (Synostose). Bei der postmortalen Untersuchung kam eine kleine ventrale Meningomyelozele zum Vorschein, die bis in eine Mittelspalte des Wirbelkörpers von $\mathrm{C} 7$ reichte.

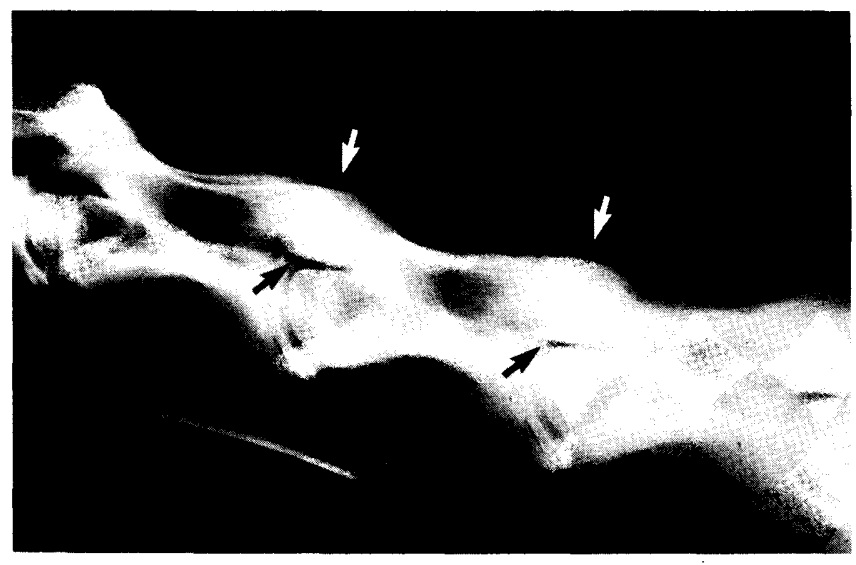

Abb. 12: Seitenansicht der Wirbel des mittleren Halsbereiches eines ataktischen 2 Jahre alten Vollblutfohlens. Die Wirbelstenose von C 4 und $C 5$ (weiße Pfeile) ist verbunden mit kurzen Wirbelbogenwurzeln und tiefgewölbten Gelenkfortsätzen, die das Lumen der Foramina intervertebralia verringern (schwarze Pfeile). Rückenmarkskompression wurde bei $\mathrm{C} 3$ zu C 4 und C 4 zu C 5 festgestellt.

xation im Bereich der Gelenkverbindung zwischen $C 2$ und C 3 oder C 3 und C 4 können beim Pferd mit einem großen sagittalen Mindestdurchmesser ohne klinische Bedeutung bleiben (Abb. 8).

Bei der Beurteilung von Röntgenaufnahmen der Halswirbelsäule ist es wichtig, nicht nur die einzelnen Wirbel zu untersuchen, sondern den Hals als Ganzes zu betrachten, ebenso wie die Form des Wirbelkanals, die Ausrichtung der Wirbelkörper, die Größe und Form der Epiphysenfugen, die Regelmäßigkeit der intervertebralen Artikulationen und die Größe der Foramina intervertebralia.

Kalkige Einlagerungen im Weichteilgewebe, d. h. als Folge einer intramuskulären Injektion, sollten nicht mit pathologischen Veränderungen der Wirbel verwechselt werden.

\section{Röntgenologische Abnormitäten}

\section{Angeborene Abnormitäten der Halswirbelsäule}

Mißbildungen der Wirbel sind selten. Obgleich Steifheit und Verdrehungen des Halses sowie Ataxie schon kurz nach der Geburt bemerkt werden, sind manche Schäden

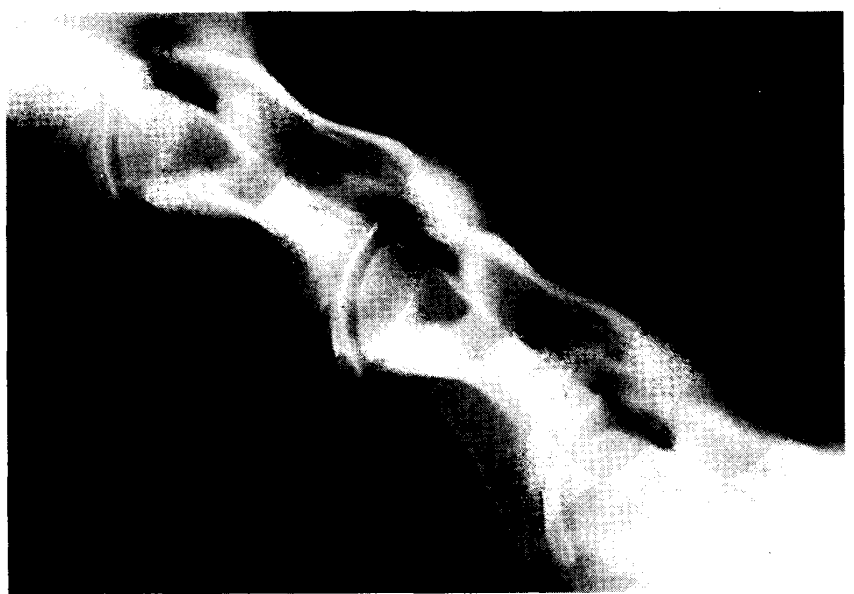

Abb. 13: Seitenansicht der mittleren Halsregion eines ataktischen 7 Monate alten Vollblutfohlens. Die kaudalen Epiphysen von C3 und C 4 sind erweitert.

über mehrere Monate klinisch inapparent, bis das Rückenmark in Mitleidenschaft gezogen wird.

Die häufigste Abweichung von der Norm betrifft das Hinterhauptsbein und die ersten beiden Halswirbel und ist als atlantookzipitale Achsenfehlstellung bekannt. Diese Art der Läsion tritt am häufigsten bei Araberpferden auf, wo eine Familienhäufigkeit besteht, aber sie erscheint auch bei anderen Rassen. Die Wirbeldefekte umfassen Verknöcherung derselben miteinander und verschiedenartige Fehlstellungsformen, die symmetrisch (Abb. 10) oder asymmetrisch sein können. An einer Wirbelkörperverknöcherung oder Synostosis können 2 oder mehrere Wirbel distal am Hals beteiligt sein. Das Fehlen von Reizkallus läßt die angeborene knöcherne von der frakturbedingten Verschmelzung unterscheiden.

\section{Entwicklungsbedingte Normabweichungen}

Die Halswirbelsäule und ihre Artikulationsflächen durchleben Umformungsvorgänge, die durch den auf sie ausgeübten biomechanischen Druck und die Knochenumsatzrate beeinflußt werden. Pferde können ein oder mehrere Halswirbeldeformationen aufweisen, wodurch Druck auf das Rückenmark ausgeübt wird, was wiederum zur Ataxie führen kann bzw. prädisponierend wirkt. Die Ätiologie und der Zeitbeginn dieser Umformungen sind nicht sicher; sie werden insgesamt als entwicklungsbedingte Normabweichungen bezeichnet.

\section{Wirbelstenose}

In diesem Fall ist der sagittale Minimaldurchmesser bei einem oder mehreren Halswirbeln von C 3 bis einschließlich C7 verringert. Es ist in vielen Fällen die Hauptursache für Rückenmarkschäden bzw. Ataxie und kommt vor allem bei jungen männlichen Vollblütern vor.

Im kaudalen Halswirbelbereich ist die Stenose generell mehr in Richtung kranialer Wirbelöffnung ausgeprägt, so daß die Öffnung des Wirbelkörpers anstatt einer rechteckigen eine mehr dreieckige Form aufweist (Abb. 7).

Eine weitere Hilfe für das Erkennen von Wirbelstenosen erfordert eine Untersuchung der Stellung der Gelenkfortsätze. Bei Vorhandensein einer Halswirbelstenose sind die 


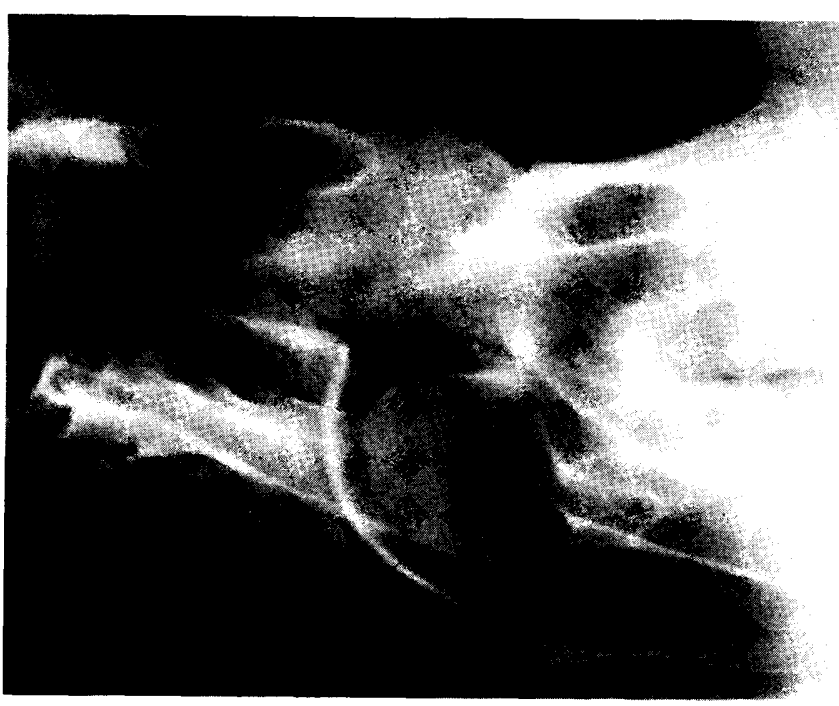

Abb. 14: Seitenansicht von $C 6$ und $C 7$ eines erwachsenen Pferdes mit Umformung und Vergrößerung der Gelenkfortsätze. Dies ist eine gewöhnliche, zufällige Normabweichung, aber möglicherweise von Si. gnifikanz die Ataxie des Pferdes betreffend. Beachte die Transposition beider ventraler Processus von $\mathrm{C} 6$ auf $\mathrm{C} 7$ und das Fehlen von Knochensäulen.

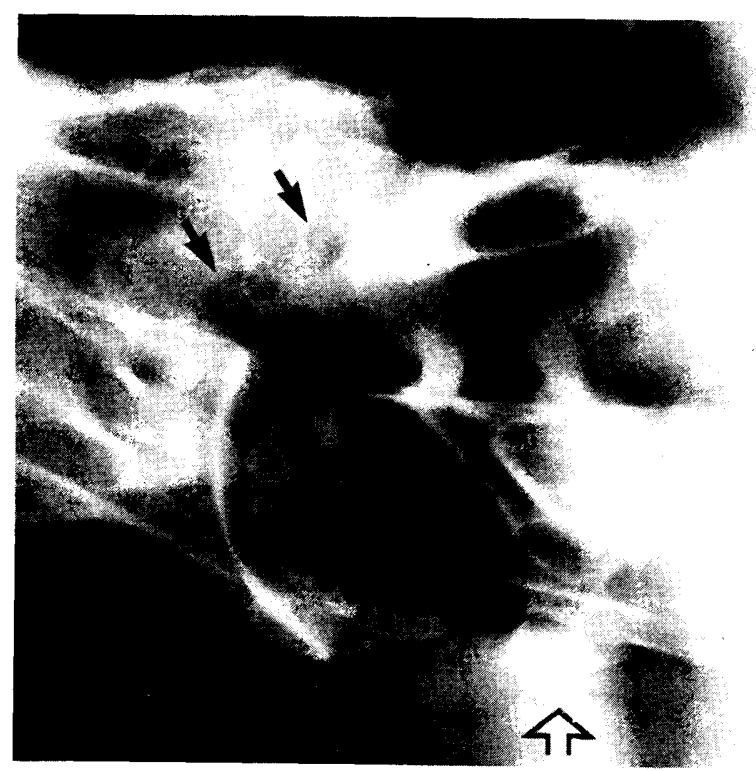

Abb. 15: Seitenansicht der kaudalen Halswirbel eines ataktischen Vollblutjährlings. Es existiert eine schwere Arthropathie bei C6 zu C7 und eine unregelmäßige Gelenkkontur. Partiell strahlendurchlässige Bezirke (schwarze Pfeile) stellen die tiefen Gruben in den Wirbelbogenwurzeln dar. Rückenmarkskompression wurde bei $C 6$ zu $C 7$ bestätigt, verbunden mit einer Synovialzyste. Ein ventraler Processus war von C6 auf $\mathrm{C} 7$ transponiert (offener Pfeil).

Wirbelbogenwurzeln (äußerer Rand des Foramen vertebrale) kurz, und der Gelenkfortsatz weist nur eine gewisse Randschärfe im Bereich des Foramen intervertebrale auf (Abb. 12).

\section{Erweiterung der kaudalen Epiphysen}

Die kaudalen Epiphysen von C2 bis C7 bilden den hintersten Bodenanteil eines jeden Foramen vertebrale. Die dorsale Erweiterung einer oder mehrerer kaudaler Epiphysen kann den Längsdurchmesser des Foramen vertebrale beträchtlich einschränken, und so können Rückenmarksquet-

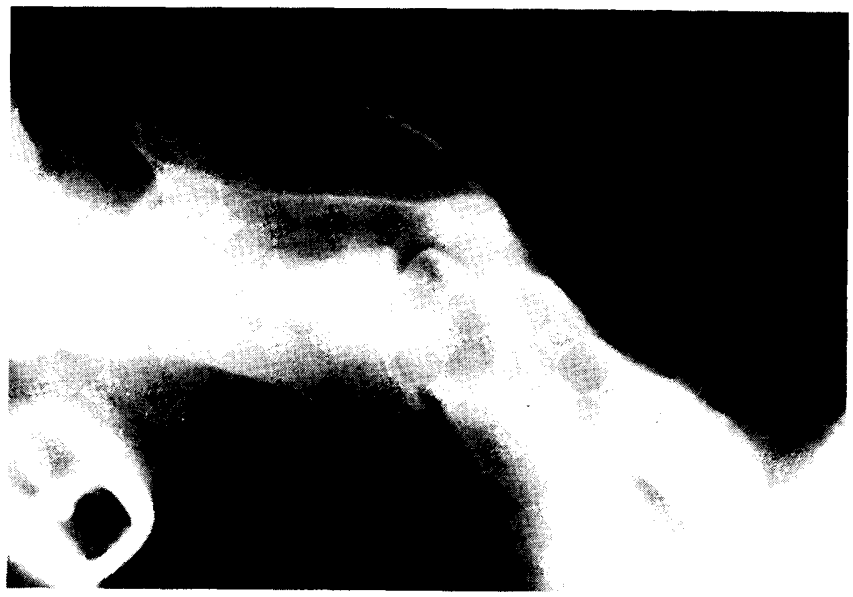

Abb. 16: Seitenansicht der kranialen Halswirbelsäule eines ataktischen 2jährigen Vollblutfohlens. Es ist eine gravierende Hyperflexion bei C2 zu C3, typisch für eine Ronney-Typ-I-Läsion, vorhanden.

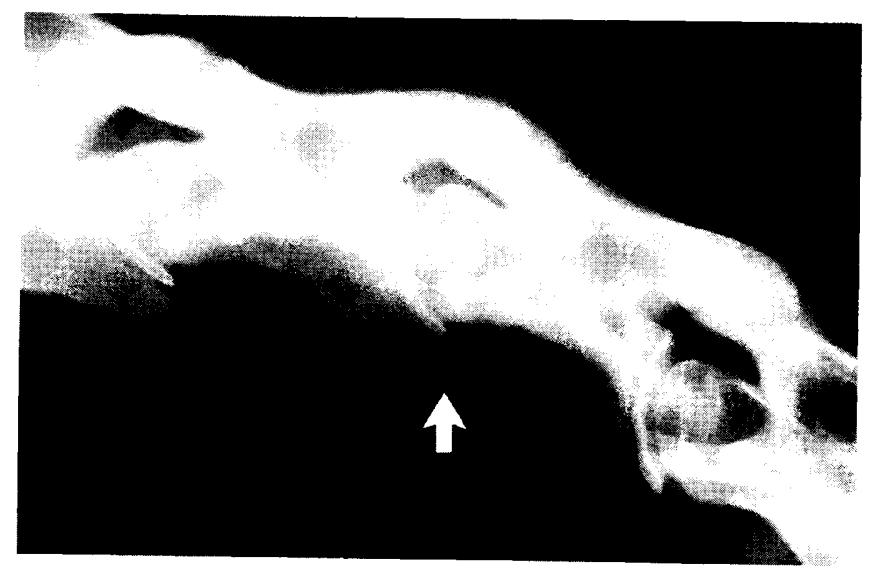

Abb. 17: Seitenansicht aus der mittleren Halsregion eines ataktıschen Vollblutjährlingsfohlens. Es ist eine Subluxation von C3 und C4 (Pfeil) mit Verringerung des sagittalen Mindestdurchmessers vorhanden. Rückenmarksquetschung in diesem Bereich wurde sicher festgestellt.

schungen und Ataxie entstehen, die normalerweise in den ersten fünf Lebensjahren zutage treten (Abb. 13).

\section{Arthropathie der Synovialgelenke des Halses}

Bei jungen Pferden besitzen die normalen Synovialgelenke ein glattes Profil. Anatomische Studien haben ergeben, daß bei ca. $50 \%$ der normal ausgewachsenen Pferde geringe einoder beidseitige Umformungen an den Artikulationsflächen von $\mathrm{C} 6$ zu C7 (Whitwell, unveröffentlichte Ergebnisse) auftreten. Dies ist oft mit einer Vergrößerung des Faserknorpels über die kraniale Begrenzung des dorsalen Wirbelbogens von $\mathrm{C} 7$ hinaus und mit einer unregelmäßigen Verbreiterung der Gelenkfortsätze verbunden. Die Veränderungen sind röntgenologisch als verschwommene Konturen erkennbar (Abb. 14).

Der Dornfortsatz von C7 kann bei Kontakt mit C 6 abgeflacht oder sogar abgebrochen werden, wenn der Hals eine Hyperextensionsstellung einnimmt. Obwohl dies normale röntgenologische Abweichungen von der Norm sind, sind sie nicht notwendigerweise von klinischer Bedeutung.

Diese Umformungsvorgänge sind jedoch Grundvoraussetzung für das Entstehen epiduraler Synovialzysten, die eine häufige Ursache für Rückenmarksquetschungen im Be- 


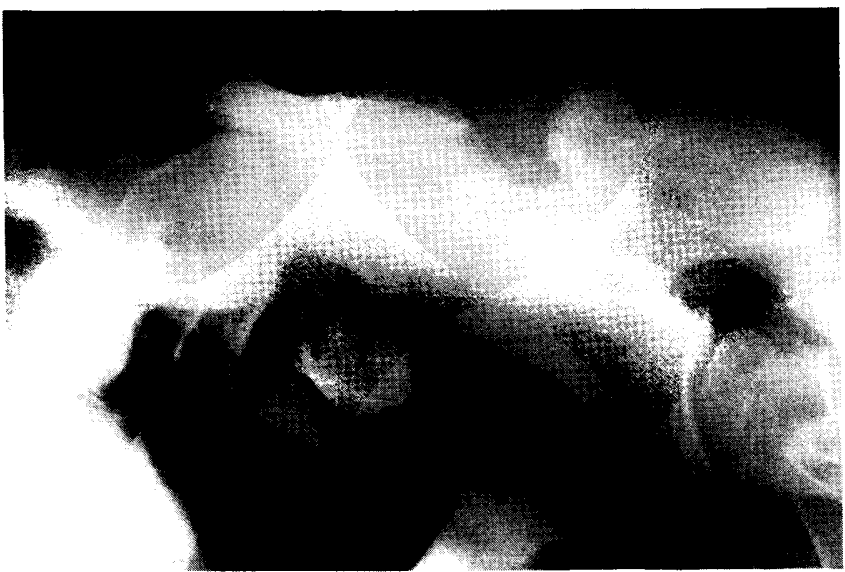

Abb. 18: Seitenansicht der kranialen Halsregion eines 9 Wochen al ten Vollblutfohlens, das Halsstarre, Schmerzen, Halsskoliose und später Ataxie aufwies, nachdem es auf einen Zaun geprallt war. Röntgenbilder, die 19 Tage später gemacht wurden, lassen eine Atlasfraktur erkennen, die durch die Knorpelfuge zwischen oberem und unterem Wirbelbogen (Pfeile) zieht und eine Einklemmung des Dens des Atlas auf einem Kondylus des Hinterhauptbeines aufweist. Das Rückenmark war, durch die Verschiebung des Atlas nach oben, gequetscht.

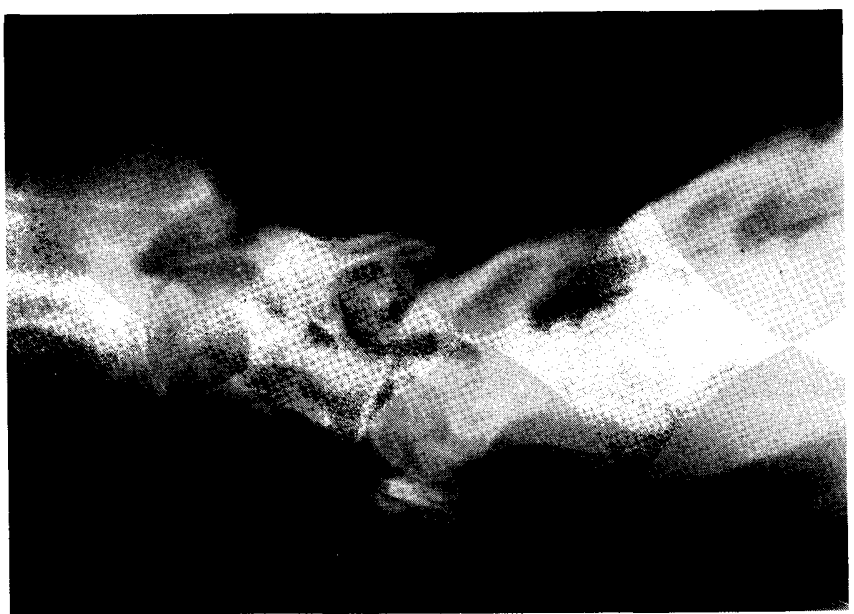

Abb. 19: Seitenansicht eines Vollblutfohlens mit „verdrehtem Hals“. Es liegen Trümmerfrakturen der Wirbelkörper und Gelenkfortsätze von C3 und C 4 vor, wodurch Stellungsanomalien entstanden sind.

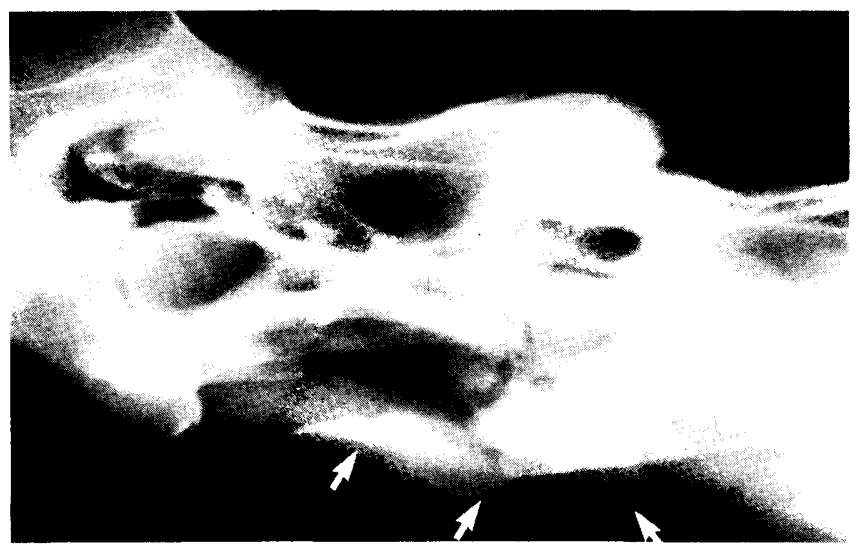

Abb. 20: Leicht schräge Ansicht von $C 2$ bis $C 4$ einer 8 Jahre alten Stute, die Genickstarre und im mittleren Halsbereich beidseits Muskelatrophie, aber keine Ataxie zeigte. Es liegt eine verschobene Fraktur im ventrokaudalen Bereich des Wirbelkörpers von C 3 vor, die Folge eines Sturzes kopfüber

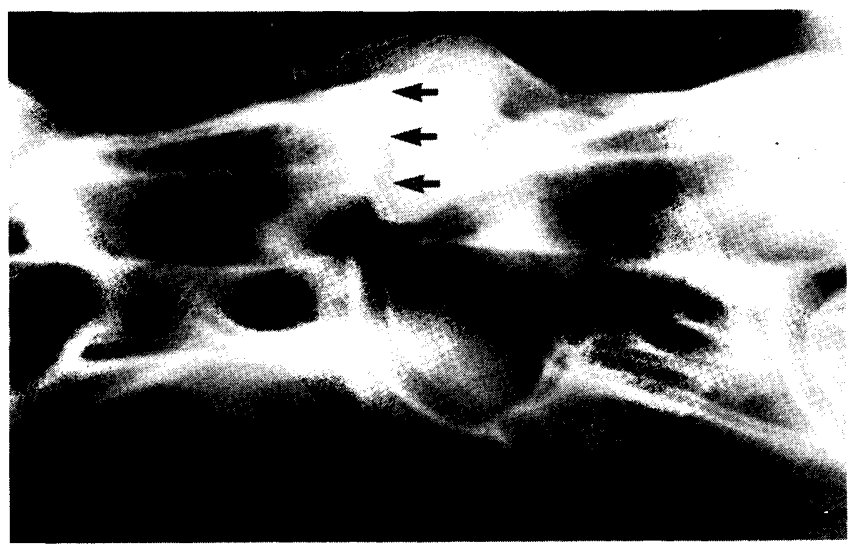

Abb. 21: Seitenansicht der kaudalen Halswirbelsäule eines ataktischen 2 Jahre alten Voliblutfohlens. Es besteht eine Frakturlinie durch die Gelenkfortsätze bei C5 und C6 (Pfeile) infolge einer Arthropathie Die postmortale Untersuchung bestätigte, daß eine Fraktur durch den linken kranialen Gelenkfortsatz von C6 bestand. Die kraniale Wirbelkörperöffnung von C6 war eingeengt, dies wurde als Hauptursache der Rückenmarksquetschung im Bereich von $\mathrm{C} 6$ zu $\mathrm{C} 7$ angesehen.

reich des Gelenkes von C6 zu C7 sind (Whitwell, 1980). Schwerwiegendere pathologische Veränderungen können einseitig oder beidseitig im Bereich der Synovialgelenke des Halses entstehen (Abb. 15 und 21). Erosionen, Fissuren und sogar pathologische Frakturen über der Gelenkoberfläche sind darin eingeschlossen. Sie werden begleitet von Gelenkerweiterung und manchmal von erhabenen periartikulären Knochenzubildungen. Gelegentlich weist die Außenfläche der Wirbelbogenwurzeln bzw. der Knochenleisten Vertiefungen in unmittelbarer Nachbarschaft eines von der Norm abweichenden Synovialgelenkes auf. Wenn die Einbuchtungen tief sind, entstehen partiell strahlendurchlässige Hohlräume (Abb. 15). Röntgenaufnahmen von beiden Seiten des Halses können hilfreich sein, die Seite festzustellen, auf der die einseitige Schädigung vorhanden ist. Dies entsteht, weil die kassettennahe Läsion sich schärfer abzeichnet und einen geringeren Vergrößerungseffekt hat als eine kontralateral angefertigte Aufnahme des Halses.

Ein häufig anzutreffender Röntgenbefund sowohl beim gesunden als auch beim ataktischen Pferd ist das Vorhandensein einer knöchernen Auftreibung auf der Unterfläche eines oder beider Processus articulares. Wenn sie stark ausgebildet ist, stellt sie einen Pfeiler dar, der den Wirbelkörper oder Wirbelbogen des kranial davor gelegenen Halswirbels beeinflußt und mit ihm ein falsches Gelenk bildet. Der "Pfeiler" verlegt teilweise das Foramen intervertebrale (Abb. 6), aber dies ist nicht generell von klinischer Bedeutung, es sei denn, die Gelenkkapsel ist medial ausgesackt und drückt auf das Rückenmark. Anatomische Studien haben ergeben, daß knöcherne Auftreibungen zunächst bei C7 vorkommen (18\% der „normalen“ männlichen Pferde haben zumindest eine Auftreibung). Sie treten aber auch bei C3 (11\%) und C5 (Rooney-Typ-II-Läsion) auf und können Rückenmarksquetschungen hauptsächlich bei Fohlen (Rooney, 1963) hervorrufen. Diese Schäden sind im seitlichen Strahlengang nicht zuverlässig sichtbar und bei dorsoventraler Aufnahmetechnik nur schwer auszumachen. Rooney (1963) beschreibt außerdem eine seltene, aber un- 


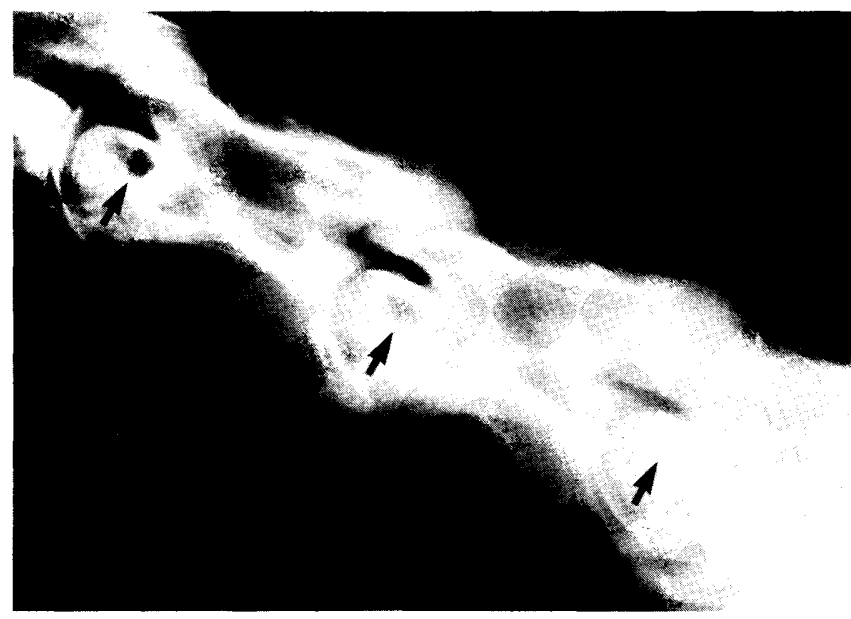

Abb. 22: Seitenansicht der Halswirbelsäule einer 5 Jahre alten Voll blutstute mit starker Halsstarre, persistierender Leukozytose und Neu trophilie und Anstieg von $\beta$-Globulinen. Es existieren partiell strahlen durchlässige Bezirke der Wirbelkörper (Pfeile), die von einem sklerotischen Saum umgeben waren. Postmortal wurde eine tuberkulöse Osteomyelitis festgestellt

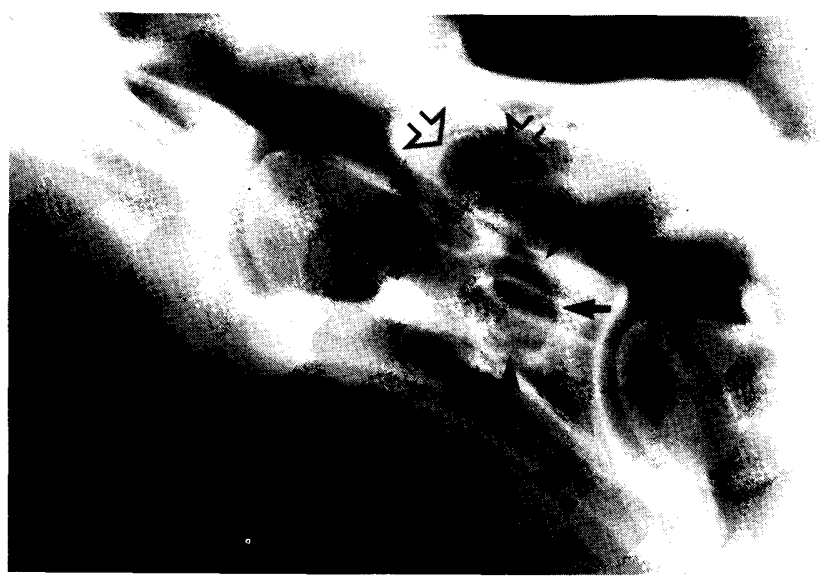

Abb. 23: Seitenansicht der kaudalen Halswirbelsäule eines 2 Jahre alten Vollblutfohlens mit Halsstarre, Schwitzfleck im linken Halsbereich, Atrophie des Musculus supraspinatus und Lahmheit, vorne links. Im Bereich der Wirbelkörper sind partiell strahlendurchlässige Bezirke (schwarze Pfeile) und in einem der Wirbelbogenwurzeln von C6 (offe ne Pfeile) als Folge ein Eindringen von neurofibromatösem Gewebe Das Pferd starb plötzlich wegen völligen Zusammenbruchs des geschwächten C6 und infolge Verletzung des Rückenmarks.

verkennbare Normvariante (Typ-I-Läsion), die nur im Gelenk von C2 zu C3 auftritt, das eine ständige Hyperflexionsstellung einnimmt, verbunden mit Fehlstellung der Gelenkfortsätze. Der Wirbelkanal ist folglich stark eingeengt, und dies wiederum führt zu Rückenmarksquetschungen. Pferde mit dieser Mißbildung haben eine normale Genickstellung, und die Röntgenaufnahme ist charakteristisch (Abb. 16) dafür.

\section{Instabilität der Wirbel bzw. Subluxation}

Eine Änderung der biomechanischen Kräfte auf die Halswirbel kann für Fehlstellungen verantwortlich sein, die manchmal zwischen benachbarten Wirbeln auftreten. Von dieser Veränderung ist zumeist die Gelenkverbindung von C 3 zu C 4 dort betroffen, wo im seitlichen Strahlengang die Subluxationsstellung des 4. Halswirbels erscheint. Sie

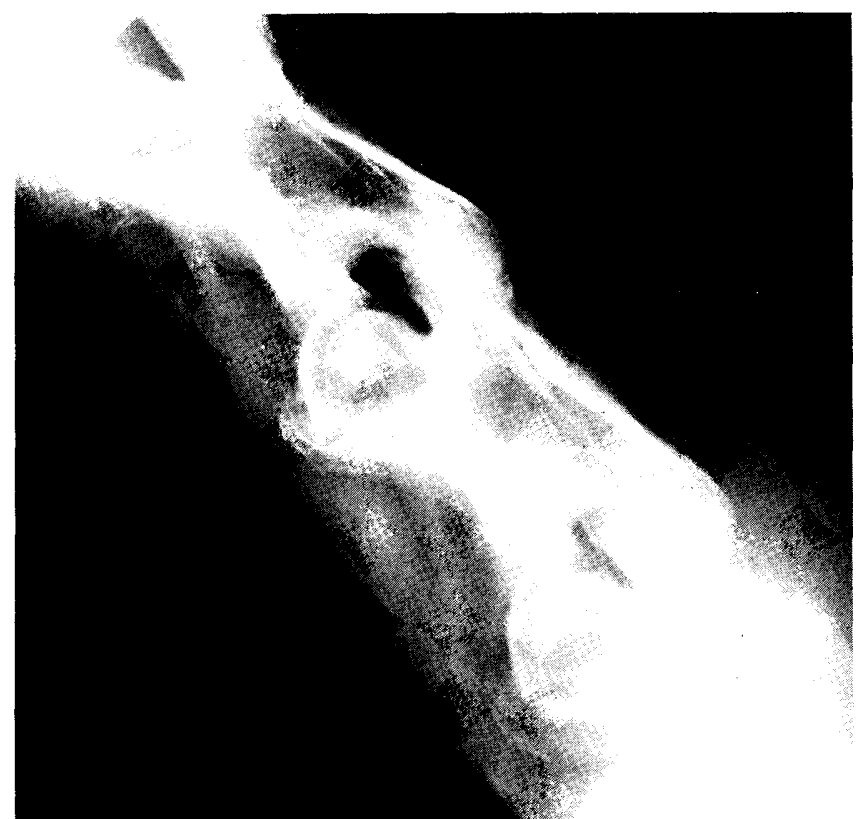

Abb. 24: Seitenansicht der mittleren Halsregion eines 18 Jahre alten Ponywallachs. Beachte die diffuse Osteoporose, die die Knochenbälkchen unnatürlich hervortreten läßt. Der Mangel an Knochendichte resuitiert aus der schlechten Differenzierung der Halswirbel auf der Unterseite mit dem umgebenden Weichteilgewebe.

ist oft schon in Ruhehaltung sichtbar und wird beim Abbiegen des Halses betont. Die Subluxationsstellung ist häufig von einer oder mehreren der oben beschriebenen Entwicklungsanomalien begleitet, und gemeinsam führen sie zu einer örtlich signifikanten Verkürzung des Längsdurchmessers des Wirbelkanals (Abb. 17). Dies hat häufig Rükkenmarksquetschungen zur Folge. Gelegentlich gibt es eine wechselseitige Hyperextension bei vermehrt kaudaler Artikulation, was in Ruhestellung zur Zickzackanordnung der Halswirbel führt.

\section{Frakturen}

Halswirbelfrakturen sind unmittelbare Folge eines Traumas oder erfolgen sekundär nach vorhergehendem pathologischem Geschehen. Ihre Bedeutsamkeit hängt von der Frakturlinienführung und der Kallusbildung sowie von der Verlagerung der Fragmente ab. Bei jungen Pferden kann eine Fraktur die unverschmolzenen Knorpelfugen durchqueren. Dies geschieht bevorzugt beim Atlas von Fohlen, wo die Knorpelfugen relativ ungeschützt sind (Abb. 18). Frakturen, die den das Foramen vertebrale umgebenden Knochen betreffen (Abb. 19), führen häufig zu Rückenmarksschäden. Frakturen der Gelenkflächen und/oder der Gelenkfortsätze können zu chronischen Schmerzen des Halses oder zu Steifheit führen.

In beiden Fällen ist eine vorsichtige Prognose zu stellen. Frakturen an anderer Stelle (z. B. Querfortsätze, Epiphysenfugen) haben eine wesentlich günstigere Prognose. Eine Impressionsfraktur des Wirbelkörpers kann von einem Sturz kopfüber herrühren. Ein dreieckiges Knochenbruchstück aus dem ventralen Bereich der kranialeren Anteile, der beiden eingeklemmten Wirbelkörper, ist verschoben (Abb. 20). Es kann als röntgenologische Normabweichung ohne klinische Bedeutung abheilen. 
Pathologische Frakturen können so zu einer Arthropathia deformans der synovialen Wirbelgelenke führen. Normalerweise verschieben die Frakturenden sich nur wenig, aber sie hinterlassen röntgenologisch eine partiell strahlendurchlässige Linie (Abb. 21). Wenn die Fraktur mit einer Ataxie verbunden ist, rechtfertigt dies nur eine sehr schlechte Prognose. Eine Fraktur des Dornfortsatzes von C7 kann zu einer ernsthaften Arthropathie von C6 zu C7 führen.

\section{Infektionsbedingte Osteomyelitis}

Systemische Infektionen (z. B. Salmonella typhimurium, Rhodococcus equi, Escherichia coli, $\beta$-hämolisierende Streptokokken) werden bei normalem oder traumatisiertem, in Auflösung befindlichem Knochen oftmals im Bereich der Wirbelkörper vorgefunden. Örtlich begrenzte, partiell strahlendurchlässige Gebiete stellen sich bei Osteomyelitis röntgenologisch dar, wobei viele Fohlen sterben, bevor osteosklerotische Knochenumbauprozesse stattfinden können. Bei älteren Pferden kann die Ausdehnung einer chronischen Weichteilentzündung oder systemischen granulomatösen Infektion (z. B. Tuberkulose) ebenfalls zur Osteomyelitis führen, wobei sie sich klinisch als Steifheit im Halsbereich darstellt. Meist sind die Wirbelkörper betroffen, woraus partiell strahlendurchlässige Bezirke unterschiedlicher Form und Größe resultieren, die von osteosklerotischen Knochenzonen umgeben sind (Abb. 22).

\section{Neoplasien}

Gewebsneubildungen der Halswirbel sind selten. Ungleichmäßige Strahlendichte der Wirbelkörper, die einen diffus verschwommenen Eindruck hinterlassen, sind für Neopla-

\section{Literatur}

Mayhew, I. (1978): Congenital Occipitoatlantoaxial Malformations in the Horse. Equine Vet. J. 10, 103-113.

Mayhew, I., Whitlock, R., und De Labunta, A. (1978): Spinal Cord Disease in the Horse. Cornell Vet. 68, Suppl. 6, 44-68.

Rantanen, N. W., Gavin, P. R., Barbee, D. D., und Sande, R. D. (1981): Ataxia and Paresis in Horses. Part II: Radiographic and Myelographic Examination of the Cervical Vertebral Column. Cont. Educ. 3, 161-171.

Rooney, J.R. (1963): Equine Incoordination I. Gross Morphologie. Cornell Vet. 53, 411-422.

Schebitz, H., und Wilkens, H. (1978): Atlas of Radiographic Anatomy of the Horse. 3rd edn., Paul Parey, Berlin und Hamburg, 43-49.

Withwell, Katherine E. (1980): Causes of Ataxia in Horses. Vet. Rec. Suppl. In Prac. 2, 17-24.

Wir danken Frl. Janet Butler für die Zusammenarbeit bei der Vorbereitung der Fotos für diese Arbeit. Dank gilt auch den vielen sien des Knochenmarks verdächtig (z. B. Lymphosarkomatose, Plasmazelltumor). Sie können zu Osteomalazie und spontanen Wirbelfrakturen führen. Neoplasien (und Mißbildungen des Nervensystems) können heimtückischen Druck auf die Wirbel ausüben. Daraus resultieren glatte Verformungen des Knochens, um den erweiterten Gewebestrukturen Platz zu schaffen. Die Neurofibromatose verursacht eine Erweiterung der Foramina transvera und intervertebralia der Wirbelkörper und -bögen (Abb. 23).

\section{Altersabhängige Normabweichungen}

\section{Spondylosis des Halses}

Kleine spondylarthrotische Fortsätze werden mitunter als Zufallsbefund auf der Ventralfläche der Wirbelkörper angetroffen, eng benachbart zu einer oder mehreren zentralen Gelenkverbindungen. Es wurde dabei keine tiefgreifende, klinisch bedeutsame Schädigung gesehen.

\section{Diffuse Osteoporose der Wirbel}

Das Fehlen von Knochensubstanz gipfelt im Verlust der Strahlendichte, zunehmender Kontrastierung zwischen Knochensubstanzverlust und Kortikalis sowie einem stärker hervortretenden Bälkchenmuster (Abb. 24). Osteoporose wird gewöhnlich bei älteren Pferden zufälligerweise gefunden, die wegen anderer Probleme geröntgt werden. Die damit zunehmende Zerbrechlichkeit der Halswirbel macht sie für Frakturen anfällig, die durch geringfügige Traumatisierung ausgelöst werden können. Osteoporose tritt außerdem bei Pferden mit Cushing-Syndrom oder mit sekundären neoplastischen Ablagerungen im Halswirbelbereich auf.
Mitarbeitern, die die Fälle der Animal-Health-Trust-Forschung zukommen ließen, ohne die diese Arbeit nicht hätte geschrieben werden können.

\author{
Sue Dyson \\ Equine Clinical Unit \\ Animal Health Trust \\ Balaton Lodge \\ Newmarket, Suffolk CB8 7DW \\ England
}

Übersetzt und veröffentlicht mit freundlicher Genehmigung

der British Equine Veterinary Association 